A small percentage of patients were administered drugs from all medication groups: hospice 17\%, community $13 \%$ and hospital $4 \%$. The number of patients administered drugs from each category varied across care setting but more patients required benzodiazepines and opioids compared to anti-secretories or anti-emetics.

Conclusion The use of anticipatory medication varies across care settings. This may be related to the average complexity of patient in each care setting. Universally there are trends in which groups of medications are used more/less often with opioids and benzodiazepines more commonly administered. Limitations include missing data and only recording medication use in the last three days of life; we will collect data for the last week of life in a repeat audit. Further community data is needed to guide a regional review including number of doses required and what medications were left at home following death.

\section{A CLINICAL AUDIT OF USE OF OCTREOTIDE IN THE MANAGEMENT OF INOPERABLE MALIGNANT BOWEL OBSTRUCTION AT A LARGE CANCER CENTRE}

Jennifer Palfrey, Vandana Vora. Guy's and St Thomas' Hospital

\subsection{6/spcare-2020-PCC.216}

Background Octreotide is a somatostatin analogue used to reduce gastric and intestinal secretions therefore helping to improve symptoms of nausea and vomiting. There is mixed evidence for the use of octreotide however in both local guidelines and the Palliative Care Formulary 6 it is considered second line for the treatment of vomiting and managing secretions in malignant bowel obstruction with hyoscine butylbromide as first line.

Objectives 1. To gain insight into the scale of prescribing of octreotide for inoperable malignant bowel obstruction in a tertiary cancer centre.

2. To compare the prescribing practices for octreotide in inoperable malignant bowel obstruction to those outlined in the local and national guidance.

Methods A retrospective audit of the electronic notes and online prescriptions for inpatients prescribed octreotide for inoperable malignant bowel obstruction over a one-year period. A total of 17 patients.

Audit standards:

1. The patient has been prescribed hyoscine butyl bromide prior to being prescribed octreotide

2. The patient has been started on a dose of octreotide between $300-600 \mathrm{mcg} / 24 \mathrm{hrs}$

3. The patient has not been prescribed a dose of octreotide greater than $1500 \mathrm{mcg} / 24 \mathrm{hrs}$

4. The patient has a clearly documented indication for the use of octreotide.

\section{Results}

- Only $41 \%$ patients had hyoscine butyl bromide prescribed prior to being prescribed octreotide.

- $100 \%$ patients received the recommended starting dose of octreotide.

- No patients received octreotide received a dose greater than $1500 \mathrm{mcg} / 24 \mathrm{hrs}$.

- $71 \%$ patients had a specific indication documented in the notes
Conclusions There are relatively few prescriptions for octreotide despite the audit data coming from a large cancer centre. Prescriptions generally follow the guidelines however documentation of indication and reason for divergence from the guideline needs improving.

Recommendations Consider using standardised documentation for when starting octreotide including indication, other medications concurrently prescribed, dietician involvement and NG tube placement.

\section{USE OF SUBCUTANEOUS FUROSEMIDE IN END STAGE HEART FAILURE: WHAT IS KNOWN IN THE LITERATURE?}

Abigail Reynolds. The Royal Wolverhampton NHS Trust

\subsection{6/spcare-2020-PCC.217}

Aim To assess what is known in the literature regarding the use of subcutaneous furosemide in advanced heart failure, as a foundation for further research.

Background Chronic Heart Failure is a leading cause of morbidity and mortality, and a growing public health problem. If resistance to oral diuretics develops amongst those with endstage disease, subcutaneous furosemide can be administered for symptom management in community settings, to prevent hospital admission and to honour preferred place of care and death. However, there is a lack of national consensus and use tends to occur in geographical pockets.

Method PubMed, EmCare and HDAS databases were used to look for articles with the key words 'subcutaneous furosemide', 'advanced', 'end-stage', 'heart failure', 'palliative care' and 'hospice'. The papers were the thematically analysed and a report compiled.

Results 15 articles were identified through database searches, and a further 3 relevant articles through other means. Just 2 papers involved a randomised controlled trial. All studies referred to the efficacy of the drug administered parenterally and several mentioned the research gap. Other areas addressed included side effects, benefits in community settings and economic implications.

Conclusions/My research idea As further research is required, I aim to develop a research project based on gathering evidence of the experience of health professionals and the efficacy of use of subcutaneous furosemide in end stage heart failure patients locally in the West Midlands. I intend to use an online survey tool.

\section{NALOXONE USE IN PATIENTS RECEIVING THERAPEUTIC OPIOIDS: ARE WE FOLLOWING THE GUIDANCE?}

Paul Selway, Charlotte French. Royal Albert Edward Infirmary, Wigan

\subsection{6/spcare-2020-PCC.218}

Introduction There is clear guidance that naloxone should not be used for patients approaching end of life and should be used at a lower dose and with great caution in those receiving long term therapeutic opioids. After being asked to review a terminally ill patient with a reduced level of consciousness who had received boluses of naloxone and was about to be started on a naloxone infusion, we decided to examine local prescribing practice and assess whether guidelines are being adhered to. 
Methods Data regarding naloxone prescribing was obtained for two three-month periods; prior to and following the introduction of a prescribing order set which included guidance on the indications for naloxone use and appropriate doses. The notes of all patients who received naloxone in each time period were reviewed; and only patients prescribed long term opioids for pain were included in the audit. Data collected included age, sex, type and dose of opioid, equivalent doses of oral morphine per day, dose of naloxone administered, stated indication for use, respiratory rate, oxygen saturations and time to death where applicable.

Results Prior to the intervention 15 patients were identified. All of these received inappropriately high doses of naloxone. Following the intervention, the number of patients reduced by more than $50 \%(n=7)$, however all patients still received inappropriate doses. Worryingly there were no patients in either time period who had documented evidence of respiratory depression; most clinicians cited reduced level of consciousness as the reason for administering the drug.

Conclusion This intervention may have reduced the number of inappropriate prescriptions of naloxone in this subset of patients. However, more education is required to improve knowledge around the appropriate use of this potentially harmful drug. Future training sessions are planned to include staff from the emergency department and acute medicine.

\section{CASE SERIES OF THE SAFE USE OF INTRAVENOUS IRON IN A HOSPICE SETTING}

Tom Steele, Helen Bonwick, Amara C Nwosu, Laura Chapman. Marie Curie Hospice Liverpool

\subsection{6/spcare-2020-PCC.219}

Background Anaemia is common in palliative care. Treatment of iron deficiency, if present, is recommended to improve symptoms and reduce dependency on blood transfusion. Previously, use of intravenous iron has been limited by the risk of anaphylaxis, however newer preparations have greatly improved safety profiles. Despite this, the feasibility of their use in hospices is unclear.

Methods A policy for administering intravenous iron at Marie Curie Hospice Liverpool was developed as part of a project to improve the targeted treatment of anaemia. Retrospective review of electronic patient and laboratory records was performed for patients receiving the intervention between October 2018 and July 2019. In all cases a single dose of Monofer (iron (III) isomaltoside) was given in keeping with the product literature.

Results 12 infusions were given to 10 patients. 4 were inpatients, the remainder day cases. Mild extravasation occurred in one case, leading to discolouration but no pain. There were no other adverse reactions. 30, 60 and 90-day survival was 92\%, $92 \%$ and $58 \%$ respectively. Baseline haemoglobin was $80 \mathrm{~g} / \mathrm{L}$ in 6 cases, $80-100$ in 3 and $>100$ in 3. All met recommended criteria for diagnosing iron deficiency in cancer (ferritin $<100 \mathrm{ug} / \mathrm{L}$ and transferrin saturation $<20 \%$ ). Fatigue was documented for all, alongside breathlessness in 4 and bleeding in 2 . 6 patients had received transfusions within the previous 3 months. Iron was given alone in 8 cases, none required subsequent transfusion. Transfusion was performed in addition to the iron infusion in 4 cases, 3 requiring further transfusions, although two had apparently decreased frequency to previously.
Conclusion This case series demonstrates the feasibility of using intravenous iron, within its product specification, to treat iron deficiency anaemia in a hospice setting. Research is required to confirm the efficacy and optimum targeting of this approach in palliative care populations.

\section{POLYPHARMACY IN CHILDREN AND YOUNG PEOPLE WITH LIFE-LIMITING CONDITIONS: AN OBSERVATIONAL COHORT STUDY IN ENGLAND}

Johanna Taylor, Deborah Gibson-Smith, Stuart Jarvis, Andrew Papworth, Michelle Hills, Veronica Neefjes, Lorna Fraser. University of York, Martin House Hospice

\subsection{6/spcare-2020-PCC.220}

Background/Aims Polypharmacy, which refers to taking several medications concurrently, is often appropriate for children and young people (CYP) with life-limiting conditions (LLCs) but can increase the risk of drug-drug and drug-disease interactions, medication errors and non-adherence, and cause unnecessary burden for families as they manage complex medication schedules. Despite this, little is known about polypharmacy in this population. This study aims to determine the prevalence of polypharmacy in CYP with LLCs.

Methods An observational cohort study of all CYP (age 0-19 years) with a diagnosed LLC in the Clinical Practice Research Datalink (primary care dataset in England) from 2000 to $2015(n=15,630)$. Unique prescriptions were identified and common definitions of polypharmacy were used to determine the prevalence in each year for all medications and for regular medications (those with at least 3 prescriptions in a 12 month period). Regression analyses were used to explore factors associated with an increased risk of polypharmacy.

Results In each year, approximately $30 \%$ of CYP were prescribed at least 5 unique medications, and $10 \%$ were prescribed at least 10 (medium annual average $=2$, range $=0-52$ ). When limiting polypharmacy to regular medications, 29\% were prescribed at least 2 medications per year, and $14 \%$ were prescribed at least 4 . Children with a primary respiratory, neurological, metabolic or circulatory diagnosis were at the greatest risk of polypharmacy. Having a second LLC or other co-morbidity were also risk factors. The proportion of children exposed to polypharmacy remained similar throughout the study period.

Conclusion This ongoing study shows that CYP with LLCs are exposed to high rates of polypharmacy. Workshops with families and clinicians held as part of the study revealed that primary care data are likely to underestimate polypharmacy in this population, and allow for limited exploration of important factors that influence their exposure to inappropriate polypharmacy.

\section{XEROSTOMIA AND XYLIMELTS USE IN HOSPICE INPATIENTS: USING THE INTEGRATED PALLIATIVE CARE OUTCOME SCALE (IPOS) TO EVALUATE}

Jessica Z Walding, Rosie Matt, Siwan Seaman. Marie Curie Hospice, Cardiff and the Vale

\subsection{6/spcare-2020-PCC.221}

Background Xerostomia is the subjective experience of oral dryness and is reported in up to $88 \%$ of advanced cancer patients. Despite use of mouthwashes, artificial saliva and 